\title{
Research on Combined Air Collision Prevention Technology
}

\author{
Hao Nie \\ The Aviation Maintenance NCO Academy of Air Force Engineering University, XinYang, 464000, China
}

\begin{abstract}
As number and frequency of flight is increasing, the safety of air traffic becomes important issue of concern. Currently what is used to ensure the safety of air traffic contains air traffic control (ATC), airborne collision avoidance system (TCAS) and automatic dependent surveillance technology (ADSB), which has been widely used. But they are independent of each other, and data fusion is not sharing, which will result that the track data of flight is not the optimization and the judgment of whether there is a potential safety hazard exists is not accuracy. This article made three kinds of technology into combination, using kalman filtering method to get data and track on the three ways for fusion, which gave the best estimate of the aircraft track, and improved the accuracy of air traffic situational judgment.
\end{abstract}

Keywords- ATC; TCAS; ads-b; kalman filtering; combination

\section{INTRODUCTION}

With the increasing of air traffic flow, the civil aviation industry demands higher and higher requirements for air traffic control safety technology. Air traffic control has successively experienced the air traffic control procedures for control, radar control and ADS-B based on satellite positioning control, at the same time civilian aircraft is equipped with air traffic alert and collision avoidance system II (TCAS II), but the TCAS II only provide transportation consulting (TA) and the decisionmaking consultation (RA) on the vertical direction, as well as TCAS II still has false alarm. Therefore, it is quite necessary to make combination of collision avoidance technology by using all kinds of information system for data fusion, this paper proposes a method based on kalman filtering of air tracking data fusion algorithm, to weighted fusion of data from various sources, to obtain the track which is the optimal estimate.

\section{AIR TRAFFIC CONTROL}

The main purpose of air traffic control is to provide all kinds of information and conflicting information for the aircraft to fly safely. The study of procedural control and radar control has been very mature and the safety of its interval standard has been verified in the air traffic control work.

\section{A. Procedural Control}

In procedural control, the only way to make the aircraft under control by phone calls between procedural controllers and pilots. The plane position, height, speed and heading, is all report to the procedural controller by the pilot, and controller has to do further evaluation of the air, then informs the pilot should fly after heading, height since the moment.
After a long period of procedural control, people find that this kind of control method has many disadvantages: it is not clear to know the state of the plane at the time, which is from calculate the estimation; The calculation is also slow and not precise; It also has a lot of restrictions on air flight, which is not conducive to increasing the flight flow in the airspace. Therefore, the importance of procedural control is gradually weakening.

\section{B. Radar Control}

Radar control from ground radar display can show the number of aircraft, aircraft flight basic state, the aircraft position, at the same time, the screen can also show the aircraft's code as well as the airline, while the controllers can observe these information, will make reasonable deployment of the aircraft.

The radar control method includes primary radar and secondary radar which is used the most. The primary radar mainly use the reflection echo of electromagnetic wave to obtain the target's oblique distance and azimuth information. Secondary radar is different, it is using airborne transponder to answer signal response that comes from the ground to detect target aircraft positioning, access code distance, azimuth, elevation and plane, as well as emergency information, etc.

In the development and application process, the secondary radar gradually highlights its advantages over primary radar, mainly including: the secondary radar system eliminates the interference of ground objects and meteorological clutter. The height information is provided by the aircraft radio altimeter and it is more accurate. The azimuth accuracy is higher than primary radar; ATC coded messages can distinguish military from civilian aircraft, while also providing warning information. Because of the above advantages, secondary radar plays an important role in many aspects, such as ATC, identification and tracking targets.

\section{TCAS ANTI-COLLISION MODEL}

TCAS II (Traffic Alert and Collision Avoidance System II, referred as TCAS II) is a stand-alone air Traffic Collision Avoidance System, the system sending signals asks the ATC transponder of the plane around, and by receiving ATC transponder response signal to get other aircraft height, distance and azimuth information, which can determine the aircraft relative motion processing, and provides the vertical determination of alarm by correct preventive measures. 


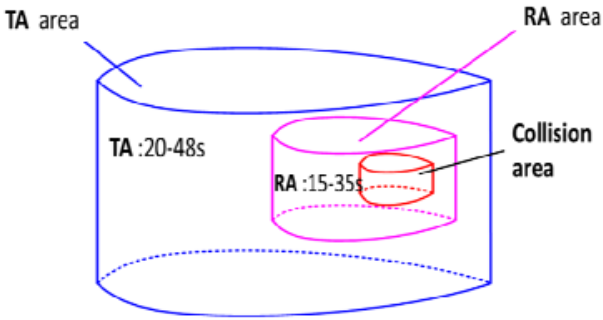

FIGURE I. AREA MODE OF TCAS II

Although the international civil aviation organization (ICAO) has make rules that large manned aircraft must install TCAS II system, with the increase of utilization rate, more and more drawbacks of TCAS II has exposed: 1 . The information source is limited, because the TCAS II height information is from secondary radar transponder using signal $\mathrm{C}$ model, but there is inconsistent with the actual height and $\mathrm{C}$ model, which increases the possibility of false alarm, and it is one of the important factors leading to the accident; 2 . TCAS II can only provide transportation consulting (Traffic Advisory, TA) and the decision-making consultation on the vertical direction (Resolution Advisory, A), confined to a vertical dimension. Currently most civilian aircraft is limited in a fixed flight on route, in an emergency situation, avoid Suggestions "climb" or "down" still have the possibility of collision with other aircraft, this position of the "dilemma" is the TCAS II limits; 3.There are blind spots for application of TCAS II. Because the TCAS II positioning accuracy is poor, so when the aircraft is on the ground, TCAS II is unable to work.

\section{THE BASIC PRINCIPLE OF ADS-B}

ADS-B technology is currently a new generation of surveillance technology that has a higher performance and a wider range of surveillance than secondary radar. ADS-B is to locate the plane four dimensional location information (longitude, latitude, altitude and time) and other necessary accessory parameters derived from airborne systems, and broadcast the data automatically, in order to complete the track of aircraft flight dynamic monitoring.

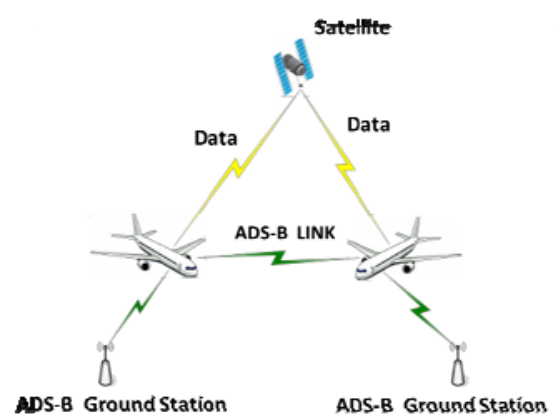

FIGURE II. COMPONENT DIAGRAM OF ADS-B

ADS-B is based on the satellite system. Signal emitted by the satellite, travel though the earth's ionosphere and troposphere, received and corrected by receiver, which in the process is influenced by many factors. According to their sources, error is generally divided into: Error associated with the satellite; Error related to signal transmission; Error associated with the receiver. These errors are usually calculated from the distance between the target and the satellite, and the distance error is given.

\section{COMPOSITE ANTI-COLLISION TECHNOLOGY BASED ON DATA FUSION}

As all kinds of aviation safety technology has some problems, we consider to put the collected various data into fusion, which can not only improve the efficiency of data processing but also the accuracy and reliability of the whole system. In this paper, a distributed fusion method is proposed for the high real-time data processing speed of civil aircraft.

\section{A. Structure of Data Fusion}

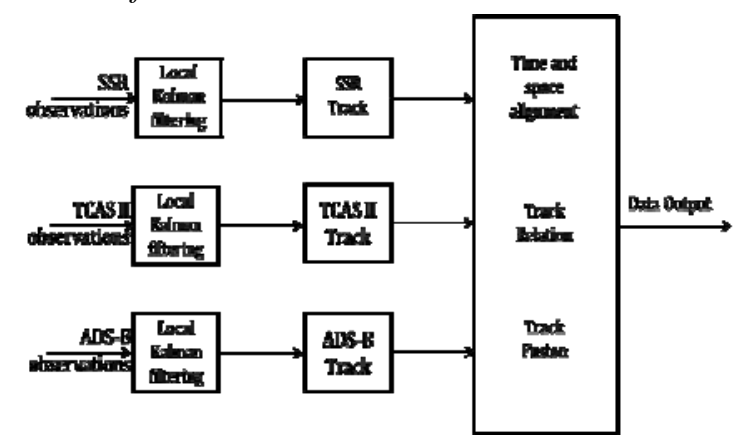

FIGURE III. STRUCTURE OF FUSION

In this fusion structure, each sensor gets their respective path of the observed information, and then data is put into filtering processing, which will output their own local track. After secondary radar, ADS-B and TCAS II local track formation is formed , they are transmitted to the fusion center, which includes "space and time alignment", "track correlation processing" and "track fusion", finally the current system of track global optimal state estimation is provided.

\section{B. Algorithm of Data Fusion}

Firstly, the data of the respective observations is filtered, and the optimal estimation criterion of Kalman filtering is linear minimum variance estimation. The state equation and measurement equation are as follows:

$$
\begin{array}{r}
X(K)=\varphi(K) X(K-1)+\omega(K-1) \\
Y(K)=H(K) X(K)+V(K)
\end{array}
$$

$X(K)$ is the system state vector of $\mathrm{k}$ moment, $\varphi(K)$ is the system state transfer matrix , $\omega(K-1)$ is the system noise, $Y(K)$ is the measurement vector of the system state for $\mathrm{k}$ moment, $H(K)$ is the measurement matrix, $V(K)$ is the measurement noise. 
Secondary radar, the TCAS II system and ADS-B system using the local filter estimate the optimization of the local state $X_{i}(K), \mathrm{i}=1,2,3,1$ represents the state of the secondary radar, 2 represents the TCAS II status, 3 represents the state of ADS-B. Based on the current statistical model, the ith local estimates of the kalman filtering and estimate covariance is respectively:

$$
\begin{gathered}
X_{i}(K+1 \mid K)=\varphi(K) X_{i}(K \mid K)+\omega(K-1) \\
P_{i}(K+1 \mid K)=\varphi(K) P_{i}(K \mid K) \varphi^{T}(K)+\omega^{T}(K)
\end{gathered}
$$

After obtaining the new observation $Y_{i}(K+1 \mid K)$, the updated value of the filtering and the covariance is:

$$
X_{i}(K+1 \mid K+1)=X_{i}(K+1 \mid K)+K(K-1)\left[Y_{i}(K+1)-H(K+1) X_{i}(K+1 \mid K)\right]
$$

$$
P_{i}(K+1 \mid K+1)=P_{i}(K+1 \mid K)\left[1-H_{i}(K+1) X_{i}(K+1)\right]
$$

$\mathrm{K}(\mathrm{k})$ is the gain of kalman filtering,

$$
K_{i}(K+1)=P_{i}(K+1 \mid K) H_{i}^{T}(K+1)\left[H_{i}(K+1) P_{i}(K+1 \mid K) H_{i}^{T}(K+1)\right]^{-1}
$$

The data is processed by parallel operation Kalman filtering, respectively obtaining the local optimal state track $X_{S S R}(K)$, $X_{\text {TCASII }}(K)$ and $X_{A D S-B}(K)$.Then the three local optimal state is merged by using the linear weighted average method, the global optimal estimate is:

$$
\begin{aligned}
& X(K)=\lambda_{1} X_{S S R}(K)+\lambda_{2} X_{\text {TCASII }}(K)+\lambda_{3} X_{A D S-B}(K) \\
& \lambda_{1}=\frac{\max \left[\lambda_{i}\left(P_{2}\right)\right]+\max \left[\lambda_{i}\left(P_{3}\right)\right]}{\max \left[\lambda_{i}\left(P_{1}\right)\right]+\max \left[\lambda_{i}\left(P_{2}\right)\right]+\max \left[\lambda_{i}\left(P_{3}\right)\right]}
\end{aligned}
$$

$$
\begin{aligned}
\lambda_{2} & =\frac{\max \left[\lambda_{i}\left(P_{1}\right)\right]+\max \left[\lambda_{i}\left(P_{3}\right)\right]}{\max \left[\lambda_{i}\left(P_{1}\right)\right]+\max \left[\lambda_{i}\left(P_{2}\right)\right]+\max \left[\lambda_{i}\left(P_{3}\right)\right]} \\
\lambda_{3} & =\frac{\max \left[\lambda_{i}\left(P_{1}\right)\right]+\max \left[\lambda_{i}\left(P_{2}\right)\right]}{\max \left[\lambda_{i}\left(P_{1}\right)\right]+\max \left[\lambda_{i}\left(P_{2}\right)\right]+\max \left[\lambda_{i}\left(P_{3}\right)\right]}
\end{aligned}
$$

$\lambda_{i}\left(P_{1}\right), \lambda_{i}\left(P_{2}\right.$ and $\lambda_{i}\left(P_{3}\right.$ are the ith feature vectors of the secondary radar,TCASII and ADS-B.

The optimal fusion estimation of the target aircraft can be obtained by weighting the secondary radar information, TCASII information and ads-b information by the maximum eigenvalue of the estimated errors.

\section{CONCLUSION}

Against the drawback of air traffic safety management of a single measure at the present, this paper using the secondary radar information, TCASII information and ADS-B information has formed combined air collision avoidance technology, mainly using the kalman filtering and the weighted estimation algorithm for various track data processing, getting the optimal estimate track data, in order to obtain the optimal state estimation of the plane. However, as the simulation data is inadequate, this algorithm is only in the theory research stage, further efforts will be put on simulation result in the future, as well as the test accuracy and feasibility of the algorithm. New technologies are becoming more and more widely used in aircraft conflict detection, with higher positioning accuracy and more accurate calculation of reserved time, which are more favorable for air safety management.

\section{REFERENCES}

[1] MAYushen, Research of Air Traffic Warning and Anti-collision Technology Based on ADS-B[M], College of Electronic \& Information Engineering Civil Aviation University of China,2014.7.

[2] Maxime Gariel, Fabrice Kunzi,R.John Hansman. AN ALGORITHM FOR CONFLICT DETECTION IN DENSE TRAFFIC USING ADSB,30th Digtal Avionics System Conference,October16-20,2011.

[3] Qi Wang , Study and Implement of Flight Track Based on ADS$\mathrm{B}[\mathrm{M}]$,JILIN University, 2015.6

[4] ICAO. Annex 10 - Aeronautical Telecommunications-Volume IVSurveillance Radar and Collision Avoidance Systems, 5th edition. [EB/OL]. http://store1.icao.int/index.php/publications/annexes/10aeronautical-telecommunications/annex-10-volume-iv-surveillanceradar-and-collision-avoidance-systems-english-printed-11328.html, 2014. 